\title{
Direct finite element route for design-by-analysis of pressure components
}

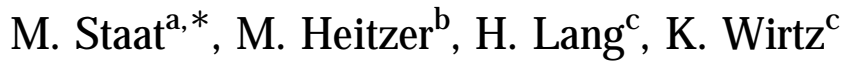 \\ aF achhochschule Aachen Div. Jülich, Ginsterweg 1, 52428 J ülich, Germany \\ ${ }^{b}$ Zentralinstitut für M athematik, F orschungszentrum J ülich $\mathrm{G} \mathrm{mbH}, 52425$ J ülich, Germany \\ ${ }^{C}$ F ramatome ANP G mbH, ND M 5, F reyeslebenstr. 1, 91056 Erlangen, Germany
}

\begin{abstract}
In the new European standard for unfired pressure vessels, EN 13445-3, there are two approaches for carrying out a Design-by-A nalysis that cover both the stress categorization method (A nnex $C$ ) and the direct route method (Annex B) for a check against global plastic deformation and against progressive plastic deformation. This paper presents the direct route in the language of limit and shakedown analysis. This approach leads to an optimization problem. Its solution with Finite Element A nalysis is demonstrated for mechanical and thermal actions. One observation from the examples is that the so-called $3 \mathrm{f}(3 \mathrm{Sm})$ criterion fails to be a reliable check against progressive plastic deformation. Precise conditions are given, which greatly restrict the applicability of the $3 f$ criterion.
\end{abstract}

Keywords: Design-by-analysis; Shakedown analysis; Progressive plastic deformation; Alternating plasticity; Shakedown criterion

\section{Introduction}

In the new European standard for unfired pressure vessels, EN 13445-3, [1], there are two approaches for a Design-by-A nalysis (DBA), that cover both the stress categorization method (Annex $C$ ) and the direct route method (A nnex B). The stress categorization method is an elastic route to the assessment of inelastic structural failure. The total elastic stress is considered to be composed of three different stress categories: primary $\sigma_{\mathrm{P}}$, secondary $\sigma_{\mathrm{Q}}$, and peak $\sigma_{\mathrm{F}}$. Admissibility is shown if the linearized stress categories do not exceed the limits in the code rules (A SM E Pressure Vessel and Boiler Code [2] or EN 13445-3 [1]). This stress categorization extrapolates limit and shakedown analysis (LISA) for simple beam and thin shell structures to complex geometries $[3,4]$ which can hardly be recommended.

The new direct route by elasto-plastic calculation in prEN 13445-3 A nnex B [1] seems to be the more promising alternative. For the time being, it is restricted to sufficiently ductile steels and steel castings below the creep range.

\footnotetext{
* Corresponding author. Tel.: +49-2461-613-437; fax: +49-2461613-133.

E-mail addresses: m.staat@fh-aachen.de (M. Staat), m.heitzer@ fz-juelich.de (M. Heitzer).
}

The paper generalizes the direct route in R ef. [4] by use of advanced LISA. The examples in Ref. [5] and in this paper show that the $3 \mathrm{f}(3 \mathrm{Sm})$ criterion [1,2] does not generally represent a conservative shakedown criterion. A new criterion is theoretically justified.

\section{Procedures for direct DBA}

The direct route calculates the design resistance (limit action) with respect to ultimate limit states of the structure. Design checks are designated by failure modes. The following ones are included in the first issue of EN 13445-3 A nnex B $[1,4]$ :

- Global Plastic Deformation (GPD), with excessive local strains and ductile rupture.

- Progressive Plastic Deformation (PD), with incremental collapse (incremental GPD, ratchetting, cyclic creep).

- Instability (I), with large displacements (buckling).

- Fatigue $(F)$, with alternating plasticity (AP) and with high cycle fatigue, i.e. fatigue with and without macroscopic plastification.

- Static Equilibrium (SE), with possible overturning and rigid body movement. 
Actions denote in Ref. [1] all thermo-mechanical quantities imposed on the structure causing stress or strain. Actions are classified by their variation in time: permanent $(G)$, variable $(Q)$, exceptional $(E)$, and operating pressures and temperatures $(p, T)$. Partial safety factors $\gamma$ are used in the GPD check for each action to obtain their design values and to define their combined design effect $E_{d}$,

$E_{d}=E_{d}\left(\gamma_{G} G, \gamma_{p} p, \gamma_{\top} T, \gamma_{Q} Q, \ldots\right)$.

With the partial safety factor $\gamma_{R}$ for the resistance $R$ of the structure, the design strength $R_{d}$, and the effects $E_{d}$ are compared in the design check

$E_{d} \leq R_{d}=\frac{R(G, p, T, Q, \ldots)}{\gamma R}$,

which is performed directly in actions with LISA. The notion of a design strength has been used in R efs. $[1,4]$ but is not used any more in the present [1].

\subsection{Material models in DBA routes}

In Refs. [1,4] perfectly plastic materials models are used for LISA in DBA. However, a closer look at the code rules shows that hardening is indirectly taken into account through the dependence of the partial safety factor on the ratio $R_{p 0.2 / t} / R_{m / 20}$ in the GPD check. Hardening can provide some additional safety margin, which could be estimated by advanced LISA with kinematic hardening material models [6]. Depending on the steel and on the analysis conditions different design strengths $f$ are used in the stress categorization route such as

$f=\min \left\{\frac{R_{p 0.2 / t}}{1.5}, \frac{R_{m / 20}}{2.4}\right\}$

for, e.g. ferritic steels [1] with $R_{p 0.2 / t}$ at design temperature $t$ and $R_{m / 20}$ at $20{ }^{\circ} \mathrm{C}$. Traditionally the GPD check has to be performed with the more conservative Tresca yield surface $\Phi_{\top}(\boldsymbol{\sigma})=R_{d}$, whereas the PD check uses the more realistic von $M$ ises yield surface $\Phi_{M}(\boldsymbol{\sigma})=R_{d}$. The numerical difficulties in FEA with the non-smooth Tresca yield surface are avoided in Refs. [1,4] by reducing the von M ises limit conservatively by $\sqrt{3} / 2$.

\section{Lower bound LISA}

Checks against failure modes GPD and PD may be directly performed by LISA based on Melan's static or lower bound theorem. Conservatively the code [1] requires the use of a perfectly plastic material model and the sidecondition that the maximum absolute value of the principal strains does not exceed $5 \%$. It could be asked if the simple perfectly plastic model allows a conservative estimation of the plastic strain accumulation during ratchetting, because all known cyclic plasticity models fail on one or more material ratchetting experiments [7]. However, it is easily demonstrated that the difference in shakedown analyses for some linear and nonlinear kinematic hardening models is smaller than could be expected from cyclic analyses with such models [6].

\subsection{Check against global plastic deformation (GPD)}

Static theorems are formulated in terms of stress. They define safe structural states leading to an optimization problem for safe monotonic and cyclic loads. The maximum safe action is the limit load (avoiding GPD) and the elastic shakedown load (avoiding PD and AP), respectively.

For the LISA approach inequality (2) is not well defined, because the design effect is a collection of incompatible design quantities: scalar temperatures $T_{d}$, vector volume forces $\boldsymbol{q}_{\mathrm{d}}$ in volume $\mathrm{V}$, and vector surface traction $\boldsymbol{p}_{\mathrm{d}}$ on the traction boundary $\partial \mathrm{V}_{\sigma}$ with unit normal vector $\boldsymbol{n}$. Let us assume that the most unfavourable actions have been combined to a single design action vector $\boldsymbol{A}_{\mathrm{d}}=\left(\boldsymbol{q}_{\mathrm{d}}, \boldsymbol{p}_{\mathrm{d}}\right)$. Then-in the sense of the GPD check-the action is admissible if the yield condition

$\Phi_{\top}(\boldsymbol{\sigma}) \leq \mathrm{R}_{\mathrm{d}}$ in $\mathrm{V}$

is satisfied with the design resistance $R_{d}$ (allowable stress) and the Tresca yield function $\Phi_{\top}$. Trivially the structure must be in static equilibrium, i.e.

$-\operatorname{div} \boldsymbol{\sigma}=\alpha_{\mathrm{s}} \boldsymbol{q}_{\mathrm{d}}$ in $\mathrm{V}, \boldsymbol{n}^{\top} \boldsymbol{\sigma}=\alpha_{\mathrm{s}} \boldsymbol{p}_{\mathrm{d}}$ on $\partial \mathrm{V}_{\sigma}$.

These conditions can be stated in words as the

Static limit load theorem: An elastic-plastic structure will not collapse (GPD) under a monotonic effect $\alpha_{s} \boldsymbol{A}_{d}$, if it is in static equilibrium and if the yield function is nowhere violated.

For each stress field $\boldsymbol{\sigma}$, which fulfills the conditions of the static theorem, $\alpha_{\mathrm{s}}$ is a safety factor, so that the load-carrying capacity of the structure is not yet exhausted if $\alpha_{\mathrm{s}} \geq 1$. Then the design check of inequality (2) is replaced by the condition $\alpha_{\mathrm{s}} \geq 1$.

$$
\begin{array}{cc}
\max \alpha_{\mathrm{s}} & \\
\text { s.t. } \Phi_{\mathrm{T}}\left[\alpha \boldsymbol{\sigma}^{\mathrm{E}}(\boldsymbol{x}, \mathrm{t})+\boldsymbol{\rho}(\boldsymbol{x})\right] \leq \mathrm{R}_{\mathrm{d}}(\mathrm{x}) & \text { in } \mathrm{V}, \\
\operatorname{div} \boldsymbol{\rho}(\boldsymbol{x})=0 & \text { in } \mathrm{V}, \\
\boldsymbol{n}^{\top} \boldsymbol{\rho}(\boldsymbol{x})=0 & \text { on } \partial \mathrm{V}_{\sigma} .
\end{array}
$$

This maximization problem is discretized by FEM and solved for monotonic time history (i.e. $\boldsymbol{\sigma}^{E}(\boldsymbol{x}, \mathrm{t})=\boldsymbol{\sigma}^{\mathrm{E}}(\boldsymbol{x})$ in (6)) effectively with optimization algorithms $[8,9]$. A large $\alpha_{\mathrm{s}}$ can be used to improve the design.

It should be clearly stated that, without restrictions, this limit load is truly independent of load history, elastic data and self-equilibrated stress (secondary stress). This statement is slightly modified if the side-condition on the maximum absolute value $(5 \%)$ of the principal strains is 
considered. Five examples in Ref. [4] have been analysed with the methods cited in Section 4, see Ref. [10]. The reduction of the limit load by the strain limit was well within the numerical differences between the FEM analyses in all cases.

\subsection{Check against progressive plastic deformation (PD)}

Strictly, only one load case can be checked by limit analysis. Design checks should be performed by shakedown analysis, if actions are time-variant. The time history of an action $\boldsymbol{A}(\mathrm{t})=(\boldsymbol{q}(\mathrm{t}), \boldsymbol{p}(\mathrm{t}), \mathrm{T}(\mathrm{t}))$ is often not well-known. It can however usually be stated that the actions vary within given amplitudes or admissible bounds. They define a convex load range $\mathscr{L}$. If $\mathrm{NV}$ is the number of independent actions $\boldsymbol{A}_{1}, \ldots, \boldsymbol{A}_{\mathrm{j}}, \ldots, \boldsymbol{A}_{\mathrm{NV}}$, varying in the bounds $\xi_{\mathrm{j}}^{-} \boldsymbol{A}_{\mathrm{j}}^{0} \leq \boldsymbol{A}_{\mathrm{j}} \leq \xi_{\mathrm{j}}^{+}$ $\boldsymbol{A}_{\mathrm{j}}^{0}$ with the reference load $\boldsymbol{A}_{\mathrm{j}}^{0}$, respectively, then all actions $\boldsymbol{A}(\mathrm{t}) \in \mathscr{L}$ can be represented by convex combination of NV vertices $\boldsymbol{A}_{\mathrm{j}}$ of $\mathscr{L}$, see Fig. 1,

$$
\begin{aligned}
& \boldsymbol{A}(\mathrm{t})=\lambda_{1}(\mathrm{t}) \boldsymbol{A}_{1}+\ldots+\lambda_{\mathrm{j}}(\mathrm{t}) \boldsymbol{A}_{1}+\ldots+\lambda_{\mathrm{NV}}(\mathrm{t}) \boldsymbol{A}_{\mathrm{NV}} \\
& \text { with } 0 \leq \lambda_{\mathrm{j}}, \quad \sum_{\mathrm{j}=1}^{\mathrm{NV}} \lambda_{\mathrm{j}}=1 .
\end{aligned}
$$

The load-carrying capacity is exhausted by enlargement of $\mathscr{L}$ with the factor $\alpha>1$ causing PD, AP or GPD. The shakedown theory analyzes only the shakedown state. The shakedown theorems answer the question, whether a structure from ductile material is plastically safe or not. Generally, a structure under a load range $\mathscr{L}$ shakes down, if a time-invariant residual stress field $\boldsymbol{\rho}(\boldsymbol{x})$ can be found such that the time-variant stress field $\boldsymbol{\sigma}(\boldsymbol{x}, \mathrm{t})=\boldsymbol{\sigma}^{\mathrm{E}}(\boldsymbol{x}, \mathrm{t})+\boldsymbol{\rho}(\boldsymbol{x})$ is statically admissible and the yield condition is fulfilled for all loads in $\mathscr{L}$; in other words
Static shakedown theorem: An elastic-plastic structure will not fail with macroscopic plasticity (PD, AP, and GPD as special case) under time variant actions in $\alpha_{\mathrm{s}} \mathscr{L}$ if a time-invariant residual stress field $\boldsymbol{\rho}$ can be found such that the structure is in static equilibrium and the yield function is nowhere and at no instance violated. Then the plastic deformation rates tend to zero.

Strictly, an independent fatigue analysis must show that the plastic dissipation is bounded. A gain one is interested in the largest factor $\alpha_{s}$, for which the structure shakes down to asymptotically elastic behaviour.

The same conditions as in the limit load theorems must be satisfied simultaneously at all times. Their examination in infinitely many instants is impossible and in addition, unnecessary. One can show that it is sufficient to satisfy the shakedown conditions only in the NV basis actions $\boldsymbol{A}_{1}, \ldots$, $\boldsymbol{A}_{\mathrm{NV}}$ of $\mathscr{L}$ since the shakedown theorems lead to convex optimization problems:

$$
\begin{aligned}
& \max \alpha_{\mathrm{s}} \\
& \text { s.t. } \Phi_{\mathrm{M}}\left(\alpha_{\mathrm{s}} \boldsymbol{\sigma}_{\mathrm{j}}^{\mathrm{E}}(\boldsymbol{x})+\boldsymbol{\rho}(\boldsymbol{x})\right) \leq \mathrm{R} \quad \text { in } \mathrm{V}, \mathrm{j}=1, \ldots, \mathrm{NV} \\
& \operatorname{div} \boldsymbol{\rho}(\boldsymbol{x})=0 \quad \text { in } \mathrm{V}, \\
& \boldsymbol{n}^{\top} \boldsymbol{\rho}(\boldsymbol{x})=0 \quad \text { on } \partial \mathrm{V}_{\sigma} .
\end{aligned}
$$

By $\boldsymbol{\sigma}^{\mathrm{E}}=\boldsymbol{\sigma}_{j}^{\mathrm{E}}$ the optimization problem of Eq. (6) is obtained with the only change that now the constraints have to be satisfied for all $\mathrm{j}=1, \ldots, \mathrm{NV}$ simultaneously. It is not sufficient to examine the critical load cases independently, because the shakedown analysis of $\mathscr{L}$ and the limit analysis of the critical load cases (vertices of $\mathscr{L}$ ) may give different results. PD is considered less critical than GPD, because it evolves over many cycles and can therefore be discovered by in-service inspections. Therefore, the ASME code [2]

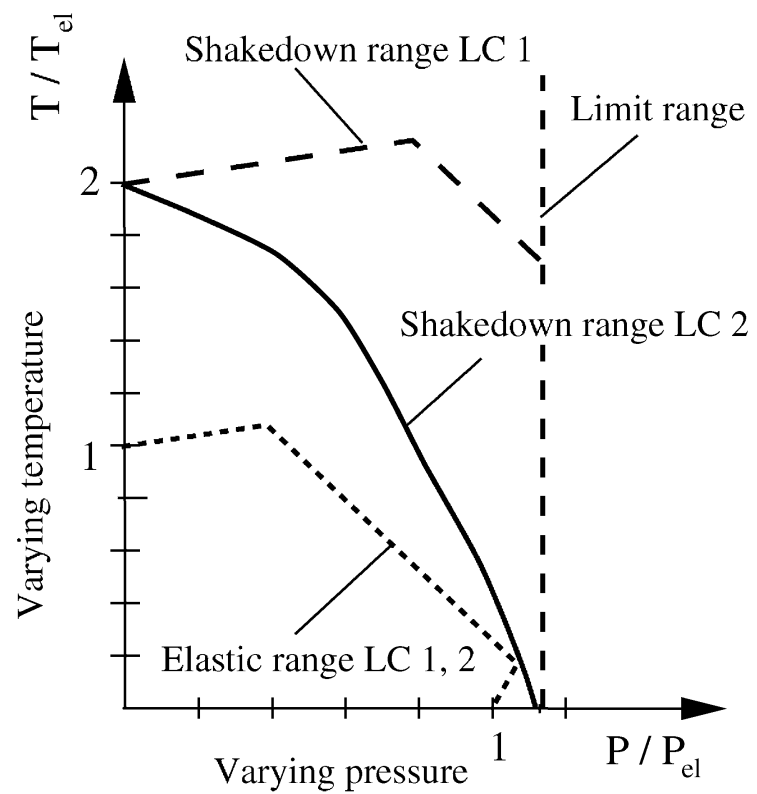

$(0,0)$
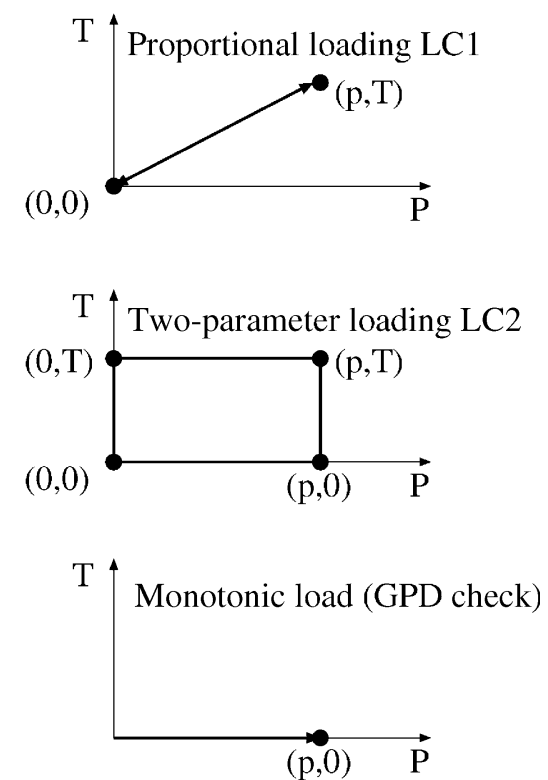

Fig. 1. Interaction diagram by FEA for a thin pipe and convex load domains $\mathscr{L}$ for pressure and temperature actions. 
and EN 13445-3 [1] do not use safety factors in the PD check.

Different to limit analysis the shakedown theorems are more complicated for hardening material. In DBA there is currently no need for such complications, because the present code requirements are restricted to perfectly plastic material $[1,4]$. It should be pointed out that different to limit analysis, shakedown analysis does not apply if the elastically calculated stress field contains singularities. The standard [1] (A nnex B.3.9.3.2) states that the check against PD can be performed for a stress-concentration-free structure, such that sharp corners have to be removed eventually from the FEM models.

\subsection{Check against alternating plasticity (AP)}

The optimization problem (7) (i.e. the PD check), includes a check against $A P$, which is accepted in the DBA manual ([4], p. 2.40), because it is conservative. The proof of shakedown is easier to perform than cyclic plastic analyses in a PD check. Shakedown analysis can distinguish between A P and PD [11], by a well known application of the shakedown theorem (see [12]). In the AP check, the yield condition $\Phi_{\mathrm{M}}\left[\alpha_{\mathrm{S}} \boldsymbol{\sigma}_{\mathrm{j}}^{\mathrm{E}}(\boldsymbol{x})+\boldsymbol{\rho}(\boldsymbol{x})\right] \leq \mathrm{R}$ for the load domain $\mathscr{L}$ with NV load vertices can be replaced by using the bounds $\xi_{j}^{+}, \xi_{j}^{-}$for the load vertices $\boldsymbol{A}_{\mathrm{j}}$ where all combinations of the signs \pm are to be accounted for (Eq. (4.39) on p. 51 of Ref. [12]):

$\Phi_{\mathrm{M}}\left[\frac{1}{2} \sum_{\mathrm{j}=1}^{\mathrm{N} V} \pm\left(\xi_{j}^{+}-\xi_{\mathrm{j}}^{-}\right) \alpha_{\mathrm{s}} \boldsymbol{\sigma}_{\mathrm{j}}^{\mathrm{E}}(\boldsymbol{x})\right] \leq \mathrm{R}$.

\section{Direct DBA by LISA}

It is the objective of shakedown analysis to demonstrate that the structure shakes down to stable elastic behaviour after few initial plastic cycles and to compute the related safety margins. The DBA M anual [4] uses two methods for shakedown analysis that do not employ standard optimization procedures: elastic compensation $([13,14])$ and deviatoric map [15]. We have implemented LISA into the general purpose FEM program PERMAS [16] employing sequential quadratic programming together with an iteration in a subspace of the space of residual stresses. This method can handle very large optimization problems [17] and has been generalized to kinematic hardening materials in Ref. [9]. FEM -based LISA may be used to compute interaction diagrams for any complex component. Such diagrams show the allowable load range for a structure and are used to decide on the design load range. For demonstration it is assumed that all partial safety factors are unity in the GPD check, i.e. $\gamma=1$. Inconsistent results are avoided by use of the von Mises yield function also for limit analysis. The analyses may be performed at computing costs of only $3-10$ linear elastic finite element analyses (FEA). Thus design variants may be compared and an optimum choice be made. A comparison with Ref. [1] is discussed in Ref. [10] for two problems with purely mechanical actions.

\subsection{Thin pipe}

A thin pipe with the ratio $t / R_{1}=0.1$ between inner radius $\mathrm{R}_{1}$ and the thickness $\mathrm{t}$ is subjected to pressure and thermal gradient. The pipe is discretized by an axisymmetric ring element with quadrilateral cross section with 4 nodes. The element is subjected to axisymmetric mechanical and thermal actions by inner pressure $p$ and by slowly varying temperatures. The outer temperature $T_{a}$ is assumed to be zero such that the thermal difference $T_{i}-T_{a}$ corresponds to the inner temperature $T_{i}$. A linear temperature distribution in the thin shell and temperature independent material data are assumed. The following load cases $\mathscr{L}$ are considered (see Fig. 1):

LC1: Pressure $p$ and temperature difference $T$ vary simultaneously with a proportionality factor (one-parameter action) with two load vertices $\mathbf{P}_{1}=(0,0), \mathbf{P}_{2}=(p, T)$.

LC2: The pressure $p$ and T vary independently (twoparameter action) with four load vertices $\mathbf{P}_{1}=$ $(p, 0), \mathbf{P}_{2}=(p, T), \mathbf{P}_{3}=(0, T), \mathbf{P}_{4}=(0,0)$.

GPD: The collapse pressure $p$ is obtained under monotonic action with only one load vertex $\mathbf{P}_{1}=(p, 0)$.

Fig. 1 shows the calculated interaction diagrams of the cases $L C 1$ and $L C 2$. The pressure and temperature actions are normalised to the yield pressure and temperature, respectively. In LC1 the shakedown factor is 2 until the pressure reaches the collapse pressure. The temperature action may have an influence on the limit load only through a change of the resistance $R(T)$. It is not a load, because the thermal stresses are residual stresses. In the case of pure mechanical loads the analytical limit pressure $\mathrm{P}_{\text {limit }}=$ $0.11 \sigma_{\mathrm{y}}$ exceeds the yield pressure $\mathrm{P}_{\text {elast }}=0.10 \sigma_{\mathrm{y}}$ by $10 \%$. The load cases $L C 1$ and $L C 2$ and their interaction diagrams differ from the classical Bree diagram (constant primary load) of a thin pipe under constant pressure and cyclic temperature.

\subsection{Pipe with non-symmetric thermal gradient}

The example consists of a pressurized closed pipe with inner radius $R_{1}$ and outer radius $R_{2}$ with $R_{2} / R_{1}=1.2$ which is subjected to thermal gradients induced by a stratification of the two-phase flow in the interior of the horizontal pipe. For the shakedown analysis five load vertices are chosen from the data set of given temperature distributions with the highest temperature $T_{\max }$. The temperature actions $T_{j}$ are applied as temperature differences $\Delta \mathrm{T}_{\mathrm{j}}=\mathrm{T}_{\mathrm{j}}-50^{\circ} \mathrm{C}$. The load domain $\mathscr{L}$ is spanned by five independent load vertices 

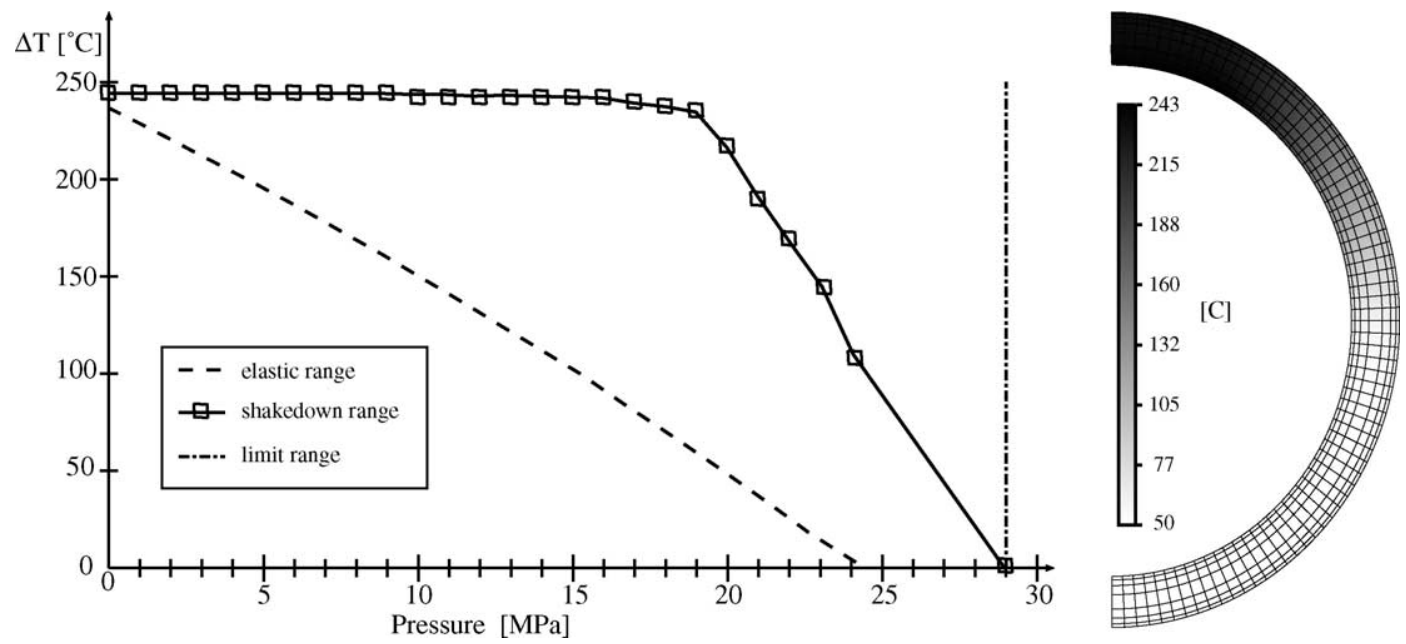

Fig. 2. Interaction diagram by FEA for a pipe with cyclic thermal gradients from stratified two-phase flow over steady internal pressure and example temperature distribution.

which makes an illustration impossible. A good fit for the temperature dependent yield stress in the given temperature range is

$\sigma_{\mathrm{y}}(\mathrm{T})=198.0-0.2^{\circ} \mathrm{C}^{-1} \cdot \mathrm{T}[\mathrm{MPa}]$.

After the first shakedown analysis the maximum temperature is estimated and the temperature dependent material data are updated. The temperature independent material data are taken at $300^{\circ} \mathrm{C}$, i.e. $\mathrm{E}=179 \mathrm{GPa}$, $\nu=0.3, \alpha_{\top}=1.7 \times 10^{-7} \mathrm{~K}^{-1}$.

Fig. 2 illustrates the highest admissible temperature differences $\Delta \mathrm{T}_{\max }=244{ }^{\circ} \mathrm{C}$ for the different constant pressures. The calculated limit pressure $29.0 \mathrm{M} \mathrm{Pa}$ is close to the exact thick pipe solution $29.1 \mathrm{MPa}$. Following the standard [1] the limit actions for PD become never effective in all examples in Ref. [4], because the GPD limits are typically much lower after reduction by division with $2 \gamma_{\mathrm{p}} \gamma_{\mathrm{R}} / \sqrt{3}$ for dominating mechanical actions. This indirect safety margin is lost in shakedown checks under dominating thermal actions, because the secondary stress $\sigma_{0}$ is not limited in the GPD check.

\section{Recommendations for the codes rules}

With the design strength $\mathrm{f}$, the $3 \mathrm{f}$-criterion $\Delta \sigma^{\mathrm{E}} \leq 3 \mathrm{f}$, see Ref. [1] (3Sm in Ref. [2]), for elastically computed total stress range $\Delta \sigma^{\mathrm{E}}=\Delta\left(\sigma_{\mathrm{P}}+\sigma_{Q}\right)$ combined with the limit load criterion for the primary membrane stress $\sigma_{\mathrm{P}_{\mathrm{m}}}$ and primary stress $\sigma_{\mathrm{p}}$ can be derived (Eq. (3))

$\Delta\left(\sigma_{\mathrm{P}}+\sigma_{\mathrm{Q}}\right) \leq 3 \mathrm{f}=2 \mathrm{R}_{\mathrm{p} 0.2}, \sigma_{\mathrm{P}_{\mathrm{m}}} \leq \mathrm{f}=\frac{2}{3} \mathrm{R}_{\mathrm{p} 0.2}$

and $\sigma_{\mathrm{P}} \leq 1.5 \mathrm{f}=\mathrm{R}_{\mathrm{p} 0.2 \text {. }}$

This is equival ent to the statement that it is necessary for the structure to shakedown elastically that the shakedown range be twice as large as the elastic range but not larger than the limit load range. It is surprising that the 3 -criterion is offered for a general shakedown check, because it seems to have its origin in a simplified argument for the shakedown check against A P only. A hysteresis loop occurs for perfect plasticity and kinematic hardening material if $\boldsymbol{\sigma}^{\mathrm{E}}>\mathrm{R}_{\mathrm{p} 02}$. One can state that A P occurs if the total strain exceeds twice the elastic strain at first yielding. Comparison with the interaction diagrams Figs. 1 and 2 however demonstrates that the use of the $3 \mathrm{f}$-criterion can hardly be recommended, because the shakedown range is typically much smaller with the exception of proportional actions in LCl. If the shakedown analysis is restricted to an AP check then twice the elastic yield is obtained as a limit also for twoparameter actions in LC2 [11]. More precisely, we can state the

Static shakedown criterion for local failure: An elastic-plastic structure will not fail locally with macroscopic alternating plasticity (AP) under time variant actions with proportional change of all actions, if the elastically computed equivalent stress range nowhere exceeds twice the initial yield stress range.

This follows immediately from the classical alternating plasticity criterion applied to proportional loading by setting $\mathrm{NV}=1, \xi_{1}^{+}=1 \xi_{1}$ in Eq. (8). On the other hand, if in the structure the PD mode is decisive the proposed criterion is invalid. In practice, it is not known in advance, which failure mode will occur in a structure. From a mathematical point of view, the AP criterion and the elastic shakedown criterion for unbounded kinematic hardening material are identical in the case of proportional loading, such that this material model is not capable of modeling incremental collapse. In Ref. [6] an example of a rotating disk under a radial temperature distribution and an angular velocity $\omega$ is given where the disk fails under proportional volume loading due 
to PD for a perfectly plastic material. For purely mechanical loading $\omega$ the shakedown factor is lower than 2, such that it is clear that this factor cannot be applied to proportional loading in general.

Therefore, $\Delta \sigma^{\mathrm{E}}=\Delta\left(\sigma_{\mathrm{P}}+\sigma_{Q}+\sigma_{\mathrm{F}}\right)$ in Eq. (9), such that the AP check

$$
\begin{aligned}
& \Delta\left(\sigma_{\mathrm{p}}+\sigma_{\mathrm{Q}}+\sigma_{\mathrm{F}}\right) \leq 2 \mathrm{R}_{\mathrm{p} 0.2}, \sigma_{\mathrm{P}_{\mathrm{m}}} \leq \frac{2}{3} \mathrm{R}_{\mathrm{p} 0.2} \\
& \text { and } \sigma_{\mathrm{P}} \leq \mathrm{R}_{\mathrm{p} 0.2}
\end{aligned}
$$

follows with peak stress $\sigma_{\mathrm{F}}$. Heuristic arguments are given by a figure in Ref. [3] or by the deviatoric map in R ef. [4]. The proof shows that there is no benefit from kinematic hardening for the safety of a structure under the conditions of the above criterion. Therefore, $3 f$ has to be replaced strictly by $2 R_{p 0.2}$ in the criterion, i.e. there should be no indirect contribution of $R_{m}$ in Eq. (3) to the design strength. The local character of the failure shows that the conditions of the criterion should include also the nonsingular peak stress $\sigma_{\mathrm{F}}$. Moreover, the criterion cannot be applied if the structure fails globally with PD, except for the case of actions that cause purely secondary stresses $\sigma_{Q}$ (purely secondary action).

Static shakedown criterion for thermal loading: An elastic-plastic structure will not fail with macroscopic plasticity (PD or AP) under proportional time variant thermal action with zero mechanical actions, if the elastically computed equivalent stress range nowhere exceeds twice the initial yield stress range.

A pplying the static shakedown criterion for local failure the shakedown load factor $\alpha_{s}$ can be at most 2. The elastic stresses $\boldsymbol{\sigma}^{\mathrm{E}}$ corresponding to proportional thermal loading are self-equilibrated stresses, such that by choosing $\boldsymbol{\rho}=$ $-\boldsymbol{\sigma}^{\mathrm{E}}$ in problem (6) it is clear that 2 is also a lower bound for $\alpha_{\mathrm{s}}$ and thus follows $\alpha_{\mathrm{s}}=2$ independent of the failure mode.

For purely secondary actions with proportional change a simple PD check requires

$\Delta \sigma_{Q} \leq 2 \mathrm{R}_{\mathrm{p} 0.2}$ and $\sigma_{\mathrm{p}}+\sigma_{\mathrm{F}}=0$.

Reducing the real GPD limit actions by division with $2 \gamma_{\mathrm{p}} \gamma_{\mathrm{R}} / \sqrt{3}$ for the above examples, the interaction diagrams demonstrate that failure by wrong application of the $3 f$ criterion may not necessarily lead to practical problems, because the GPD check rules out most critical actions.

\section{Conclusions}

The well known $3 f$ criterion can be justified for a PD check only under purely thermal actions. However, in design practice no problems are expected if mechanical actions dominate, because then the partial safety factors and the Tresca yield function make the GDP check usually more restrictive than the $3 f$ criterion. As the new direct route for DBA deals directly with the plastic failure modes, the results give better insight for the analyst into the structural behaviour under all possible mechanical or thermal actions, the safety margins, and the possibilities for design or operation improvement. LISA can be performed directly by optimization procedures implemented in an FEM code. In fact, the costs and simplicity of LISA compare favourably with standard elastic FEA. Therefore, there is little argument to retain the stress classification route with the inherent problems of that concept.

\section{Acknowledgements}

We would like to thank J.L. Zeman, Vienna Technical University, for discussing the PD check in prEN 13445-3. This work has been partly supported by the European Commission through their Brite-EuRam III Programme (Project BE 97-4547, Contract BRPR-CT97-0595).

\section{References}

[1] Unfired pressure vessel standard EN 13445, Part 3: Design, Annex $B$ Direct route for design by analysis and Annex $C$ Stress categorisation route for design by analysis. European Committee for Standardization (CEN), EN 13445-3, Issue 1, M ay 2002.

[2] ASME Boiler and Pressure Vessel Code, Section III, Rules for Construction of Nuclear Power Plant Components, Div. 1, Subsection NB, Class 1 Components, and Section VIII, Rules for Construction of Pressure V essels, Div. 1 and 2. New Y ork: The A merican Society of Mechanical Engineers; 1995.

[3] Hechmer JL, Hollinger GL. 3D stress criteria for application. New Y ork: W elding Research Council Bulletin; 1998. p. 429.

[4] Taylor N, editor. The design-by-analysis manual. 1999. Error Corrections. Available at http://info.tuwien.ac.at//AA/news/ dbal_engl.htm.

[5] Staat M. Some achievements of the European Project LISA forFEM based limit and shakedown analysis. In: Badie N, editor. Computational mechanics: developments and applications. ASM E PVP, vol. 441; 2002. p. 177- 85.

[6] Staat $M$, Heitzer $M$. The restricted influence of kinematic hardening on shakedown loads. In: Mang HA, Rammersdorfer FG, Eberhardsteiner J, editors. Fifth W orld Congress onComputational M echanics, July 7-12, Vienna, A ustria. 2002.

[7] Bari S. Constitutive Modeling for Cyclic Plasticity and Ratcheting, PhD Thesis, North Carolina State University, Raleigh, 2001.

[8] Staat M , Heitzer M. LISA a E uropean Project for FEM -based limit and shakedown analysis. Nucl Eng Des 2001;206:151-66.

[9] Staat $M$, Heitzer $M$, editors. Numerical methods for limit and shakedown analysis. NIC-series, vol. 15. Jülich: John von Neumann Institutefor Computing; 2003. A vailable at http://www.fz-juelich.de/ nic-series/volume15/volume15.html.

[10] Staat M , Schwartz M , Lang H, W irtz K, H eitzer M. Design by analysis of pressure components by non-linear optimization. In: Zeman JL, editor. Pressure vessel technology, Proceedings ICPVT-10, July 7- 10, Vienna, A ustria. 2003. 
[11] Vu DK, Y an AM, Nguyen-Dang H. A dual form for discretized kinematic formulation in shakedown analysis. Int J Solids Struct 2004;41(1):267- 77

[12] König JA. Shakedown of elastic-plastic structures. A msterdam: Elsevier; 1987.

[13] M ackenzie D, B oyle JT, et al. A method of estimating limit loads by iterative elastic analysis I, II and III. Int J Pressure Vessels Piping 1993;53(1):77- 142.

[14] Ponter ARS, Engelhardt M. Shakedown limits for a general yield condition: implementation and application for a von Mises yield condition. In: Weichert D, Maier G, editors. Inelastic analysis of structures under variable loads: theory and engineering applications. Dordrecht: Kluwer; 2000.

[15] Preiss R, Zeman JL. The Deviatoric map-a simple tool in DBA. Int J Pressure V essels Piping 1999:76:339- 44.

[16] INTES PERMAS User's reference manual. PERMAS Version 7, INTES Publication N o. 450. Stuttgart: Rev. E; 1999.

[17] Heitzer M, Staat M. FEM - computation of load carrying capacity of highly loaded passive components by direct methods. Nucl Eng Des 1999;193:349- 58. 